\title{
Dynamic Bargaining and Stability with Veto Players*
}

\author{
Vincent Anesi $^{\dagger} \quad$ John Duggan ${ }^{\ddagger}$
}

April 15, 2016

\begin{abstract}
This note examines the structure of stationary bargaining equilibria in the finite framework of Anesi (2010). The main result establishes a tight connection between the set of equilibrium absorbing points and the von Neumann-Morgestern solutions: assuming that players are patient, that the voting rule is oligarchical, and that there is at least one veto player with positive recognition probability, a set of alternatives corresponds to the absorbing points of an equilibrium if and only if it is a von Neumann-Morgenstern solution. We also apply our analysis of ergodic properties of equilibria to the persistent agenda setter environment of Diermeier and Fong (2012). We show that all equilibria are essentially pure, and we extend their characterization of absorbing sets to allow an arbitrary voting rule and by removing the restriction to pure strategy equilibira.
\end{abstract}

\section{Introduction}

Since the seminal work of Baron (1996), bargaining games with an endogenous status quo have become more and more prominent in the literature

\footnotetext{
${ }^{*}$ We are grateful to two anonymous referees of this journal for many useful comments and suggestions.

${ }^{\dagger}$ School of Economics, University of Nottingham. Email: vincent.anesi@nottingham.ac.uk.

${ }^{\ddagger}$ Department of Political Science and Department of Economics, University of Rochester. Email: dugg@ur.rochester.edu.
} 
on dynamic collective decision-making. ${ }^{1}$ In these games, each period begins with a status quo alternative inherited from the previous period, and a player is chosen randomly to propose any feasible alternative, which is then subject to an up or down vote. If the proposal is voted up, then it is implemented in that period and becomes the next period's status quo; if it is voted down, then the ongoing status quo is implemented and remains in place until the next period; this process continues ad infinitum. Anesi (2010) was the first to consider the finite framework, where the set of alternatives is finite and players have strict preferences. His main goal was to provide a noncooperative interpretation for von Neumann-Morgenstern solutions (von Neumann and Morgenstern, 1944), whose rationale in the voting context had been questioned by political scientists (e.g., McKelvey et al. 1978). Assuming patient players, he shows that given a von Neumann-Morgenstern solution $Y$ for the voting rule and a sufficiently high discount factor, there is a stationary Markovian equilibrium $\sigma$ such that the set $A(\sigma)$ of absorbing alternatives under $\sigma$ is equal to $Y$. Left open is the opposite logical direction: conditions under which given a stationary Markovian equilibrium $\sigma$, the set $A(\sigma)$ of absorbing alternatives is a von Neumann-Morgenstern solution. $^{2}$ Concentrating on pure strategy equilibria, Diermeier and Fong (2012) obtain this direction by assuming, in addition to high discount factors, that the same player proposes with probability one in every period.

The main objective of this note is to contribute further to this research program by examining the structure of (mixed-strategy) stationary Markovian equilibria in the finite framework of Anesi (2010). The analysis relies on the characterization of the ergodic properties of equilibria. Namely, we show that when the Nakamura number of the voting rule is high relative to the number of alternatives, all ergodic sets are singleton; in particular, if there is a veto player (so that the Nakamura number is infinite), then beginning from any given status quo, the equilibrium process transitions with probability one to the set of absorbing alternatives. Moreover, we show that if there is a veto player with positive recognition probability and players are patient, then starting from any given alternative, there is a unique absorbing point (which can depend on the alternative given) to which the equilibrium process transitions.

\footnotetext{
${ }^{1}$ To cite a few of the many examples, Kalandrakis $(2004,2010)$, Diermeier and Fong (2011, 2012), Battaglini and Palfrey (2012), Bowen and Zahran (2012), Duggan and Kalandrakis (2012), Nunnari (2014), Richter (2014), Baron and Bowen (2014), Zápal (2014), Anesi and Seidmann (2015) and Dziuda and Loeper (2015) all use such games.

${ }^{2}$ Anesi (2010) shows by example that, under majority voting, equilibrium absorbing sets may not be von Neumann-Morgenstern solutions.
} 
These results allow us to establish a tight connection between the set of equilibrium absorbing points and the von Neumann-Morgenstern solutions. Maintaining the assumption that players are patient and there is at least one veto player with positive recognition probability, we increase the structure of our model in two directions. First, we assume that the voting rule is oligarchical, i.e., agreement of all veto players is necessary and sufficient for a proposal to pass, so that the von Neumann-Morgenstern solution is unique. Our main result is that under these conditions, the equilibrium absorbing alternatives comprise the von Neumann-Morgenstern solution of the voting rule. Second, allowing a general voting rule, we add the assumption that there is a persistent agenda setter, i.e., a fixed player who proposes with probability one in each period. We apply our analysis of ergodic properties of equilibria to show that all equilibria are essentially pure, and we again obtain the equivalence between equilibrium absorbing points and von NeumannMorgenstern solutions. Thus, we extend Theorem 1 of Diermeier and Fong (2012) by generalizing the quota rules to an arbitrary voting rule and by removing the restriction to pure strategy equilibria.

Noncooperative foundations for von Neumann-Morgenstern solutions in political economy have been investigated in several different institutional settings, including electoral competition (Anesi 2012) and committee voting (Anesi and Seidmann 2014). In particular, Diermeier et al. (2013) consider a discrete version of the divide-the-dollar environment, in which players bargain over allocations of a private good. As we do in Section 4, these authors assume the existence of at least one veto player and obtain a characterization of stationary Markov equilibria in terms of von Neumann-Morgenstern solutions. Aside from their focus on the pure distribution setting, their result differs from ours in several other important ways: they concentrate on pure strategy equilibria, assume that only veto players can make proposals, and impose an additional refinement on equilibrium proposals (i.e., when a proposer is indifferent between proposing to change the status quo and not, she does not).

Committees with veto players, who can block proposals to move away from current policies, constitute an empirically important class of institutions (e.g., the United Nations Security Council and presidential veto power in the US Congress). An extensive body of literature has developed to explore the relationships between members' policy preferences and policy outcomes in such committees. A complete review of this literature would take us far afield, but we should explain this note's relationship to the closely 
related contribution by Nunnari (2014). He analyzes a bargaining game with an endogenous status quo in the divide-the-dollar environment with three players - one being a veto player, with an additional vote needed for passage of a proposal - and equal recognition probabilities. In this setting, he constructs a stationary Markov perfect equilibrium in which the policy implemented by the committee eventually converges to the veto player's ideal policy, irrespective of the discount factor and the initial status quo. Although Nunnari's framework is not nested with ours, his result is consistent in spirit with our result that if there is a veto player with positive recognition probability, then for any given status quo alternative, there is a unique alternative to which it is absorbed. In general, this absorbing point can depend on the alternative given, but Nunnari demonstrates that in his equilibrium, it is in fact independent of the initial alternative.

More generally, this note is also part of the growing body of research on bargaining with an endogenous status quo (cf. footnote 1). In much of this literature, the focus is either on the one-dimensional spatial model with single-peaked preferences or on distributive models in which a fixed surplus is allocated across players. With few exceptions, this work explicitly constructs classes of absorbing stationary Markov equilibria. Assuming a finite set of alternatives and veto players (or a persistent agenda setter), we obtain necessary conditions for equilibrium ergodic sets that do not rely on constructive techniques and that permit a full characterization of equilibrium absorbing points (provided that players are sufficiently patient). In a framework that assumes a general voting rule and general (finite or infinite) set of alternatives, Duggan and Kalandrakis (2012) provide conditions under which every stationary Markov perfect equilibrium determines an aperiodic transition over policies, with fast convergence from any given initial policy to an ergodic distribution (that can depend on the starting point), and this ergodicity result holds in our finite framework as well, in light of wellknown results on finite-state Markov chains. Those authors can also obtain uniqueness of the ergodic distribution, but that result relies on the presence of shocks to the status quo each period.

\section{Dynamic Bargaining Framework}

Consider the following dynamic bargaining model. The set $X$ of alternatives is finite with $|X| \geq 2$, and individuals are numbered $1, \ldots, n$. In each of an infinite number of discrete periods $t=1,2, \ldots$, a status quo $x \in X$ is given, 
and a proposer is drawn from the fixed distribution $\left(\rho_{1}, \ldots, \rho_{n}\right)$, where $\rho_{i} \geq 0$ for each $i$ and $\sum_{i=1}^{n} \rho_{i}=1$. The selected individual makes a proposal $y \in X$, and all players then simultaneously vote to accept or to reject the chosen proposal. If the group $C$ of individuals who accept belongs to the collection $\mathcal{D}$ of decisive groups, then the outcome for the current period is $z^{t}=y$; and otherwise, if $C$ is not decisive, then $z^{t}=x$ is the outcome for the current period. In both cases, the current outcome $z^{t}$ becomes the status quo in the next period, where the process is repeated. Assume the voting rule $\mathcal{D}$ is nonempty and monotonic. It is collegial if there is some individual who belongs to every decisive group and has a veto, i.e., the intersection $\bigcap \mathcal{D} \equiv \bigcap_{C \in \mathcal{D}} C \neq \emptyset$ is nonempty, and we refer to such an individual as a veto player. The rule is oligarchical if in addition the set of veto players is itself decisive, i.e., $\bigcap \mathcal{D} \in \mathcal{D}$, in which case a coalition is decisive if and only if it contains all veto players.

Each individual $i$ has a stage utility function $u_{i}: X \rightarrow \Re$ such that for all distinct $x, y \in X$, we have $u_{i}(x) \neq u_{i}(y)$. Define the dominance relation $\succ$ on $X$ such that for all $x, y \in X$, the relation $x \succ y$ holds if and only if $\left\{i: u_{i}(x)>u_{i}(y)\right\} \in \mathcal{D}$. A von Neumann-Morgenstern solution (or $v N M$ solution) is a set $S \subseteq X$ satisfying both internal stability (for all $x, y \in S$, $\neg(x \succ y)$ ) and external stability (for all $x \notin S$, there exists $y \in S$ with $y \succ x)$. Note that when the voting rule is oligarchical, the dominance relation $\succ$ is transitive, so that the vNM solution is unique and consists of the maximal elements of $\succ$. Given a sequence $\mathbf{z}=\left(z^{1}, z^{2}, \ldots\right)$ of outcomes, the payoff to player $i$ is the normalized discounted utility

$$
U_{i}(\mathbf{z})=(1-\delta) \sum_{t=1}^{\infty} \delta^{t-1} u_{i}\left(z^{t}\right),
$$

where $\delta \in[0,1)$ is a common discount factor. We extend payoffs to probability distributions over such sequences via expected utility. The status quo in period 1 is an exogenously given alternative $x^{0}$.

The above elements define a dynamic game, and we focus on subgame perfect equilibria in stationary Markov strategies. Specifically, a stationary Markov strategy for player $i$ is a pair of mappings $\sigma_{i}=\left(\pi_{i}, \alpha_{i}\right)$ such that $\pi_{i}: X \times X \rightarrow[0,1]$ and $\alpha_{i}: X \times X \rightarrow[0,1]$, where:

- $\pi_{i}(x, y)$ is the probability that player $i$ proposes $y$ given status quo $x$,

- $\alpha_{i}(x, y)$ is the probability that player $i$ accepts alternative $y$ if it has been proposed and the status quo is $x$. 
We term $\pi_{i}$ the proposal strategy and $\alpha_{i}$ the voting strategy of player $i$, and we let $\sigma=\left(\sigma_{1}, \ldots, \sigma_{n}\right)$ denote a profile of stationary Markov strategies. A proposal (resp., voting) strategy is pure if for all $x, y \in X$, we have $\pi_{i}(x, y) \in\{0,1\}$ (resp., $\alpha_{i}(x, y) \in\{0,1\}$ ). Let $\alpha(x, y)$ be the probability that $y$ passes if proposed given status quo $x$, i.e.,

$$
\alpha(x, y)=\sum_{C \in \mathcal{D}}\left(\prod_{i \in C} \alpha_{i}(x, y)\right)\left(\prod_{i \notin C}\left(1-\alpha_{i}(x, y)\right)\right) .
$$

Given such a profile $\sigma$, let $P(\cdot \mid \sigma)$ denote the stochastic transition process engendered by $\sigma$, so that

$$
P(x, y \mid \sigma)=\sum_{i} \rho_{i} \pi_{i}(x, y) \alpha(x, y)
$$

is the probability that next period's outcome is $y$ given that the outcome in the current period is $x$. Then $P(x, Y \mid \sigma)=\sum_{y \in Y} P(x, y \mid \sigma)$ is the probability that next period's outcome belongs to $Y$ given current outcome $x$. In general, define $P^{1}(\cdot \mid \sigma)=P(\cdot \mid \sigma)$, and given $t \geq 2$, let $P^{t}(\cdot \mid \sigma)$ be the $t$-step transition defined by

$$
P^{t}(x, y \mid \sigma)=\sum_{z \in X} P^{1}(x, z \mid \sigma) P^{t-1}(z, y \mid \sigma),
$$

so that $P^{t}(x, \cdot \mid \sigma)$ gives the distribution over outcomes $t$ periods in the future, given outcome $x$ in the current period.

The expected discounted payoff, or dynamic payoff, starting from outcome $x$ in a given period for player $i$ is

$$
V_{i}(x \mid \sigma)=(1-\delta)\left[u_{i}(x)+\delta \sum_{t=1}^{\infty} \delta^{t-1} \sum_{z \in X} u_{i}(z) P^{t}(x, z \mid \sigma)\right] .
$$

Of course, this dynamic payoff is the unique solution to the recursion

$$
V_{i}(x \mid \sigma)=(1-\delta) u_{i}(x)+\delta \sum_{y \in X} P(x, y \mid \sigma) V_{i}(y \mid \sigma) .
$$

A stationary Markov profile $\sigma$ is a stationary bargaining equilibrium if proposals and votes are optimal at all histories; that is, if (i) for all $x \in X$ and all $i \in\{1, \ldots, n\}, \pi_{i}(x, \cdot)$ puts positive probability on solutions to

$$
\max _{y \in X} \alpha(x, y) V_{i}(y \mid \sigma)+(1-\alpha(x, y)) V_{i}(x \mid \sigma),
$$


and (ii) for all $x, y \in X$ and all $j \in\{1, \ldots, n\}$, we have $\alpha_{j}(x, y)=1$ if $V_{j}(y \mid \sigma) \geq V_{j}(x \mid \sigma)$, and we have $\alpha_{j}=0$ if $V_{j}(y \mid \sigma)<V_{j}(x \mid \sigma)$. Note that the optimality condition (ii) on voting strategies incorporates the refinement that players do not cast stage-dominated votes. Moreover, the condition assumes deferential voting strategies, so that a player votes for a proposed alternative if indifferent between acceptance and rejection. Because of this assumption, existence of a stationary bargaining equilibrium does not follow from known existence results for Markov perfect equilibria in stochastic games. Nevertheless, existence is not an issue: our first theorem does not require the deferential voting restriction; the others assume that $\mathcal{D}$ is collegial and that the discount factor is high, in which case results by Muto (1984) and Anesi (2010) imply that the game possesses a stationary bargaining equilibrium. $^{3}$

A set $Y \subseteq X$ of alternatives is invariant under $\sigma$ if for all $x \in Y$, we have $P(x, Y \mid \sigma)=1$, and it is ergodic if it is minimal among invariant sets according to set inclusion. We let $\mathcal{E}(\sigma)$ denote the collection of ergodic sets under $\sigma$. An alternative $x$ is absorbing if $P(x, x \mid \sigma)=1$, or equivalently, $\{x\}$ is ergodic. If there is some $t$ such that $P^{t}(x, y \mid \sigma)>0$, then $y$ is reachable from $x$. Let $A(x \mid \sigma)$ be the set of absorbing points that are reachable from $x$, and let $A(\sigma)$ be the set of absorbing points of $\sigma$, i.e., $A(\sigma)=\bigcup_{x \in X} A(x \mid \sigma)$.

These concepts can be reformulated in graph-theoretic terms. Define the graph of $\sigma$, denoted $\Gamma(\sigma)$, as follows: for all $x, y \in X$, we have $x \Gamma(\sigma) y$ if and only if $P(x, y \mid \sigma)>0$. Let $\Gamma^{1}(\sigma)=\Gamma(\sigma)$, and for each $t=2,3, \ldots$, define $\Gamma^{t}(\sigma)$ as follows: for all $x, y \in X$, we have $x \Gamma^{t}(\sigma) y$ if and only if there exists $z \in X$ such that $x \Gamma(\sigma) z \Gamma(\sigma)^{t-1} y$. The transitive closure of $\Gamma$, denoted $\Gamma^{\infty}(\sigma)$, is defined as $\Gamma^{\infty}(\sigma)=\bigcup_{t=1}^{\infty} \Gamma^{t}(\sigma)$. Then $Y$ is ergodic if and only if for all $x, y \in Y$, we have $x \Gamma^{\infty}(\sigma) y$ and $y \Gamma^{\infty}(\sigma) x$; an alternative $x$ is absorbing if and only if for all $y \in X, x \Gamma(\sigma) y$ implies $y=x$; and $y$ is reachable from $x$ if and only if $x \Gamma^{\infty}(\sigma) y$.

It is well-known that from any outcome $x$, the equilibrium Markov chain eventually leads to an ergodic set with probability one. To formalize this

\footnotetext{
${ }^{3}$ The former shows that there exists a (unique) vNM solution if $\mathcal{D}$ is collegial; the latter that a stationary bargaining equilibrium exists if there is a vNM solution. To be precise, Anesi (2010) assumes that $\mathcal{D}$ is proper (i.e., the complement of any decisive group in $\mathcal{D}$ does not belong to $\mathcal{D}$ ), which is implied when $\mathcal{D}$ is collegial. Moreover, he also assumes that all players have positive recognition probabilities; but brief inspection of his argument reveals that the result holds if one assumes instead (as we do) that there is at least one veto player with positive recognition probability.
} 
claim, let $P_{\infty}(x, Y \mid \sigma)=\liminf P^{t}(x, Y \mid \sigma)$. Then for all $x$, we have

$$
P_{\infty}(x, \bigcup \mathcal{E}(\sigma) \mid \sigma)=1
$$

so that with probability one the set $\bigcup \mathcal{E}(\sigma) \equiv \bigcup_{E \in \mathcal{E}(\sigma)} E$ is entered from $x$ and remains in that set. In graph-theoretic terms, for all $x \in X$, there exist an ergodic set $Y$ and an element $y \in Y$ such that $x \Gamma^{\infty}(\sigma) y$.

\section{Absorbing Alternatives}

Our first result establishes that when the Nakamura number of the bargaining game is large relative to the set of alternatives, every ergodic set is a singleton, the lone element being an absorbing alternative. To begin, we define the Nakamura number of the voting rule, denoted $\mathcal{N}(\mathcal{D})$, in two cases. First, in case the rule is non-collegial, let

$$
\mathcal{N}(\mathcal{D})=\min \{|\mathcal{G}| \mid \mathcal{G} \subseteq \mathcal{D} \text { and } \bigcap \mathcal{G}=\emptyset\} .
$$

In words, $\mathcal{N}(\mathcal{D})$ is the size of the smallest collection of decisive coalitions having empty intersection. Second, in case the rule is collegial, the set $\mathcal{N}(\mathcal{D})$ is equal to the cardinality of the integers. It is known that when the number of players is either three or strictly greater than four, the Nakamura number of majority rule is three. In general, for a quota rule with quota $q,{ }^{4}$ the Nakamura number is

$$
\mathcal{N}(\mathcal{D})=\left\lceil\frac{n}{n-q}\right\rceil
$$

so it becomes arbitrarily high when both the number of players is large and the quota is large relative to $n$. Note that when $\mathcal{D}$ is collegial, the Nakamura number is infinite, so that the assumptions of the following result are automatically fulfilled.

Theorem 1: Assume $|X|(|X|-1)<\mathcal{N}(\mathcal{D})$, and consider any stationary bargaining equilibrium $\sigma$. For every $Y \subseteq X, Y$ is ergodic if and only if there is an absorbing alternative $x$ such that $Y=\{x\}$.

\footnotetext{
${ }^{4}$ We say $\mathcal{D}$ is a quota rule if there is a natural number $q \in\left[\frac{n+1}{2}, n\right]$ such that $\mathcal{D}=\{C \mid$ $|C| \geq q\}$ consists of all groups satisfying the quota $q$.
} 
Proof: One direction is obvious. For the other, suppose toward a contradiction that there is an ergodic set $Y$ with $|Y|=k \geq 2$, and enumerate the elements as $y_{1}, \ldots, y_{k}$. For each $h=1, \ldots, k$, let

$$
Y_{h}=\left\{z \in Y \backslash\left\{y_{h}\right\} \mid P\left(y_{h}, z \mid \sigma\right)>0\right\}
$$

denote the alternatives that occur with positive probability given status quo $y_{h}$, and enumerate the elements of this set as $z_{h, 1}, \ldots, z_{h, \ell_{h}}$. For each $h=1, \ldots, k$ and each $\ell=1, \ldots, \ell_{h}$, there exists $C_{h, \ell} \in \mathcal{D}$ such that for all $i \in C_{h, \ell}$, we have

$$
V_{i}\left(z_{h, \ell} \mid \sigma\right) \geq V_{i}\left(y_{h} \mid \sigma\right) .
$$

Since $|Y| \leq|X|$ and $\left|Y_{h}\right| \leq|X|-1$, we have $|Y|\left(\max _{h}\left|Y_{h}\right|\right)<\mathcal{N}(\mathcal{D})$, so by assumption there exists $i \in \bigcap_{h=1}^{k} \bigcap_{\ell=1}^{\ell_{h}} C_{h, \ell}$. Let $m$ be a solution to $\max _{h \in\{1, \ldots, k\}} V_{i}\left(y_{h} \mid \sigma\right)$, so that $y_{m}$ maximizes player $i$ 's dynamic payoff over $Y$, i.e., $V_{i}\left(y_{m} \mid \sigma\right)=\max _{h=1, \ldots, k} V_{i}\left(y_{h} \mid \sigma\right)$. Then for all $\ell=1, \ldots, \ell_{m}$, we must have

$$
V_{i}\left(z_{m, \ell} \mid \sigma\right)=V_{i}\left(y_{m} \mid \sigma\right) .
$$

Thus, all of the alternatives that occur with positive probability following $y_{m}$ also maximize player $i$ 's dynamic payoff. Since $Y$ is ergodic, this argument in fact implies that for all $h=1, \ldots, k$, we have $V_{i}\left(y_{h} \mid \sigma\right)=V_{i}\left(y_{m} \mid \sigma\right)$, so that the dynamic payoff of player $i$ is constant on $Y$, and we can denote this by $\bar{V}$. But choosing any $y_{h}$ and $z_{h, \ell}$, we then have

$$
\begin{aligned}
(1-\delta) u_{i}\left(y_{h}\right)+\delta \bar{V} & =V_{i}\left(y_{h} \mid \sigma\right) \\
& =V_{i}\left(z_{h, \ell} \mid \sigma\right) \\
& =(1-\delta) u_{i}\left(z_{h, \ell}\right)+\delta \bar{V},
\end{aligned}
$$

which implies $u_{i}\left(y_{h}\right)=u_{i}\left(z_{h, \ell}\right)$, a contradiction. We conclude that for every ergodic set $Y$, we have $|Y|=1$, so that there is an absorbing alternative $x$ such that $Y=\{x\}$.

Q.E.D.

The next example illustrates the result in the case of two alternatives and majority rule, i.e., $\mathcal{D}=\{C \subseteq N|| C \mid>n / 2\}$. Because the Nakamura number of majority rule is equal to three if $n \neq 4$ and to four if $n=4$, it is always strictly larger than $|X|(|X|-1)=2$, and the premise of Theorem 1 always holds in this case.

Example 1: Let the set of alternatives be $X=\{x, y\}$, and assume majority rule. It is readily checked that for every stationary Markov strategy 
profile $\sigma$ and every player $i$, there is $\kappa>0$ (which depends on $\sigma$ ) such that $V_{i}(x \mid \sigma)-V_{i}(y \mid \sigma)=\kappa\left[u_{i}(x)-u_{i}(y)\right]$. Therefore, in any stationary bargaining equilibrium, voters who prefer $x$ to $y$ accept proposal $x$ given status quo $y$ and reject proposal $y$ given status quo $x$ with probability one; the same holds in reverse for those who prefer $y$ to $x$. This in turn implies that if a majority of players prefer $x$ to $y$ then, conditional on being recognized to make a proposal, those players (successfully) propose $x$ with probability one when the status quo is $y$, and they are indifferent between proposing $x$ or $y$ when the status quo is $x$, as a proposal to change $x$ to $y$ would be rejected. Hence, in every stationary bargaining equilibrium $\sigma$, we have $A(x \mid \sigma)=\{x\}$, and we have $A(y \mid \sigma)=\{x\}$ if at least one of the players who prefer $x$ to $y$ has a positive recognition probability, while $A(y \mid \sigma)=\{y\}$ otherwise. $^{5}$

Theorem 1 does not apply in the majority-rule case if there are more than two alternatives; cycles can arise and ergodic sets with multiple elements can be supported in equilibrium as Example 2, below, illustrates. But for a large set of players and quota rules with higher quotas, the result does apply. As noted above, when the voting rule $\mathcal{D}$ is collegial, the Nakamura number takes an infinite value, so the conditions of Theorem 1 are satisfied.

Example 2: Let $n=3, X=\{x, y, z\}$, and suppose that the players' utilities and discount factor satisfy the following inequalities:

$$
\begin{aligned}
& (3-\delta)^{-1}\left[(3+\delta) u_{1}(y)-2 \delta u_{1}(x)\right]<u_{1}(z)<u_{1}(y)<u_{1}(x) \\
& (3-\delta)^{-1}\left[(3+\delta) u_{2}(x)-2 \delta u_{2}(z)\right]<u_{2}(y)<u_{2}(x)<u_{2}(z) \\
& (3-\delta)^{-1}\left[(3+\delta) u_{3}(z)-2 \delta u_{3}(y)\right]<u_{3}(x)<u_{3}(z)<u_{3}(y)
\end{aligned}
$$

We further assume that $\mathcal{D}$ is majority rule, so that there is no vNM solution. Moreover, the three players have the same recognition probability, i.e., $\rho_{i}=1 / 3$ for each $i=1,2,3$. Under these assumptions, there is a (pure strategy) stationary bargaining equilibrium such that: given status quo $x$, players 2 and 3 propose and accept $y$, whereas player 1 maintains the status quo; given status quo $y$, players 1 and 2 propose and accept $z$, whereas player 3 maintains the status quo; and given status quo $z$, players 1 and 3 propose and accept $x$, whereas player 2 maintains the status quo. (We provide the precise details of the equilibrium construction in the supplementary appendix.) The induced Markov chain on $X$ is depicted in Figure

\footnotetext{
${ }^{5}$ If exactly $n / 2$ players prefer $x$ to $y$, then it immediately follows from the equilibrium voting strategies above that proposals to change status quo alternatives must be rejected in any stationary bargaining equilibrium $\sigma$, so that $A(x \mid \sigma)=\{x\}$ and $A(y \mid \sigma)=\{y\}$ in this case. Equilibrium ergodic sets are again singleton.
} 
1, where arrows represent transitions, labeled by the transition probabilities and the coalitions of players who support the transitions. Observe that there is no absorbing alternative and that the only ergodic set is the entire set of alternatives $X$.

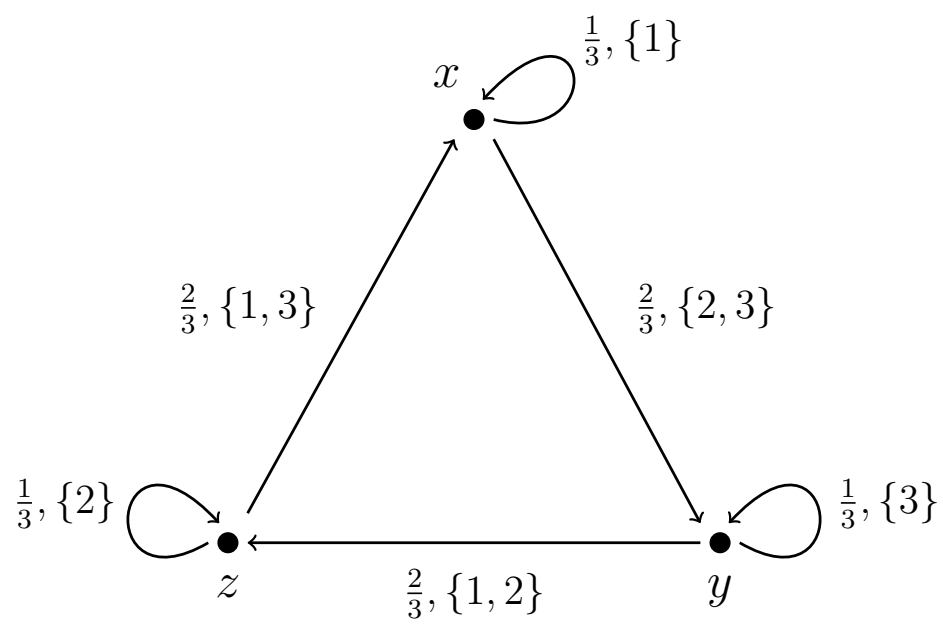

Figure 1: Equilibrium Markov chain with a Condorcet cycle

An important implication of Theorem 1 for a stationary bargaining equilibrium $\sigma$ is that for each alternative $x$, we have $A(x \mid \sigma) \neq \emptyset$. This in turn implies that beginning from any given alternative, the equilibrium Markov chain eventually transitions to an absorbing alternative with probability one.

\section{Bargaining Equilibria and vNM-Solutions}

We now increase the structure imposed on the analysis by considering the case of patient players and assuming that there is at least one veto player with positive recognition probability; in particular, Theorem 1 applies and ergodic sets are therefore singleton. The following result establishes that for any given alternative, there is in fact a unique alternative (which may depend on the alternative given) to which it is absorbed.

Theorem 2: Assume that there is at least one veto player with positive recognition probability, i.e., there exists $i \in \bigcap \mathcal{D}$ with $\rho_{i}>0$. Then there ex- 
ists $\bar{\delta} \in(0,1)$ such that for all $\delta \in(\bar{\delta}, 1)$, all stationary bargaining equilibria $\sigma$, and all $x \in X,|A(x \mid \sigma)|=1$.

Proof: By Theorem 1, we know that for all $x \in X$, we have $A(x \mid \sigma) \neq \emptyset$. To deduce a contradiction, suppose there are a sequence of discount factors $\left\{\delta^{k}\right\}$ converging to one and corresponding stationary bargaining equilibria $\left\{\sigma^{k}\right\}$ such that for each $k$, there exist $x_{k}$ with $\left|A\left(x_{k} \mid \sigma^{k}\right)\right| \geq 2$. Then $\left\{\Gamma\left(\sigma^{k}\right)\right\}$ is the corresponding sequence of equilibrium graphs. Since $X$ is finite, we can go to a subsequence (still indexed by $k$ ) on which these alternatives and graphs are constant, and henceforth we write $x=x_{k}$ for the given alternative and $\Gamma=$ $\Gamma\left(\sigma^{k}\right)$ for the equilibrium graph. Abusing notation slightly, let $A=A\left(\sigma^{k}\right)$ denote the set of absorbing alternatives of $\sigma^{k}$, and let $A(y)=A\left(y \mid \sigma^{k}\right)$ denote the absorbing alternatives reachable from any alternative $y$; these sets are constant along the sequence, and we have $|A(x)| \geq 2$. Let player $i$ be a vetoer with $\rho_{i}>0$, and let $w$ minimize the stage payoff of player $i$ among absorbing alternatives reachable from $x$, i.e., $u_{i}(w)=\min _{y \in A(x)} u_{i}(y)$. Let $\left\{y_{1}, y_{2}, \ldots, y_{m}\right\}$ be a path from $x$ to $w$, so that

$$
x=y_{1} \Gamma y_{2} \cdots \Gamma y_{m-1} \Gamma y_{m}=w,
$$

and let $y_{\ell}$ be the highest indexed alternative such that $\left|A\left(y_{\ell}\right)\right| \geq 2$, and note that $\ell<m$. Since player $i$ is a vetoer, we have

$$
V_{i}\left(y_{\ell+h} \mid \sigma^{k}\right) \geq V_{i}\left(y_{\ell+h-1} \mid \sigma^{k}\right)
$$

for all $k$ and all $h=1, \ldots, m-\ell$. This implies that $V_{i}\left(y_{\ell} \mid \sigma^{k}\right) \leq V_{i}\left(w \mid \sigma^{k}\right)$ for all $k$, and thus we have

$$
\liminf _{k \rightarrow \infty} V_{i}\left(y_{\ell} \mid \sigma^{k}\right) \leq u_{i}(w)
$$

By construction, there exists $z \in A\left(y_{\ell}\right) \backslash\{w\}$, so that $u_{i}(z)>u_{i}(w)$. Then there exist $z_{1}, \ldots, z_{h} \in X$ such that

$$
y_{\ell}=z_{1} \Gamma z_{2} \ldots \Gamma z_{h-1} \Gamma z_{h}=z .
$$

Thus, for all $k$, there exist $C_{1}^{k}, \ldots, C_{h-1}^{k} \in \mathcal{D}$ such that for all $r=1, \ldots, h-1$ and all $j \in C_{r}^{k}$, we have $V_{j}\left(z_{r} \mid \sigma^{k}\right) \leq V_{j}\left(z_{r+1} \mid \sigma^{k}\right)$. It follows that if player $i$ proposes $z_{r+1}$ given status quo $z_{r}$, the proposal will pass with probability one. ${ }^{6}$ Note, moreover, that the probability that $i$ is recognized as proposer is $\rho_{i}>0$. Since player $i$ 's equilibrium proposal strategy in equilibrium

\footnotetext{
${ }^{6}$ This inference uses the assumption of deferential voting.
} 
$\sigma^{k}$ is optimal, the expected payoff from $\sigma_{i}^{k}$ starting from status quo $y_{\ell}$ is at least equal to the following strategy: if the status quo is $z_{j}$ for some $j=1, \ldots, h-1$, then propose $z_{h+1}$ with probability one, and vote to accept $z_{h+1}$ but reject any other proposal; and for other status quos, propose and vote as in $\sigma_{i}^{k}$. We denote the latter strategy by $\tilde{\sigma}_{i}^{k}$ and the resulting strategy profile by $\tilde{\sigma}^{k}$. Accordingly, $P^{t}\left(z_{1}, z_{h} \mid \tilde{\sigma}^{k}\right)$ is the probability that $z_{h}=z$ is reached from status quo $y_{\ell}=z_{1}$ after $t$-periods. Note that the probability that player $i$ is selected as proposer $h-1$ times in $t$ periods goes to one as $t$ goes to infinity, and thus $\lim _{t \rightarrow \infty} P^{t}\left(z_{1}, z_{h} \mid \tilde{\sigma}^{k}\right)=1$. Given any $\epsilon>0$, we can choose $t$ sufficiently high that $P^{t}\left(z_{1}, z_{h} \mid \tilde{\sigma}^{k}\right)>1-\epsilon$, and then we have

$$
\begin{aligned}
V_{i}\left(y_{\ell} \mid \sigma^{k}\right) \geq V_{i}\left(y_{\ell} \mid \tilde{\sigma}^{k}\right) \geq & \left(1-\left(\delta^{k}\right)^{t-1}\right) \min _{r \in X} u_{i}(r)+ \\
& \left(\delta^{k}\right)^{t-1}\left[(1-\epsilon) u_{i}(z)+\epsilon \min _{r \in X} u_{i}(r)\right],
\end{aligned}
$$

where the right-hand side reflects player $i$ 's payoff if $z$ is in place $t$ periods in the future and remains in place thereafter, with the worst alternative in all other cases. Taking the limit as $k \rightarrow \infty$, we see that

$$
\liminf _{k \rightarrow \infty} V_{i}\left(y_{\ell} \mid \sigma^{k}\right) \geq(1-\epsilon) u_{i}(z)+\epsilon \min _{r \in X} u_{i}(r) .
$$

Since this inequality holds for all $\epsilon>0$, we conclude that

$$
\liminf _{k \rightarrow \infty} V_{i}\left(y_{\ell} \mid \sigma^{k}\right) \geq u_{i}(z)>u_{i}(w),
$$

contradicting (2).

Q.E.D.

Theorem 2 is silent on the connections between absorbing alternatives and $\mathrm{vNM}$ solutions. It shows that given any alternative $x$, there is a unique absorbing alternative, say $y$, to which it is absorbed. In particular, if $x$ is not itself absorbing, then $y \neq x$, but Theorem 2 does not imply that $y \succ x$, and so it leaves the possibility that the set $A(\sigma)$ of absorbing alternatives may violate external stability. The following example demonstrates that the presence of a veto player is not sufficient to deliver external stability of the absorbing alternatives.

Example 3: Let the set of alternatives be $X=\{x, y, a, b\}$, let there be four players, each with recognition probability $1 / 4$, and let the voting rule be such that a coalition is decisive if and only if it contains $\{1,2\}$ and at least one other player, i.e., $\mathcal{D}=\{C \mid 1,2 \in C$ and $|C| \geq 3\}$. This voting rule makes players 1 and 2 veto players, but it is not oligarchical. Stage payoffs 
satisfy

$$
\begin{aligned}
& u_{1}(x)<u_{1}(b)<u_{1}(a)<u_{1}(y), u_{2}(x)<u_{2}(a)<u_{2}(b)<u_{2}(y), \\
& u_{3}(b)<u_{3}(y)<u_{3}(a)<u_{3}(x), \text { and } u_{4}(a)<u_{4}(y)<u_{4}(b)<u_{4}(x) .
\end{aligned}
$$

Under these assumptions, $\{x, y\}$ is the unique vNM solution. We further assume that

$$
3 u_{3}(x)+u_{3}(a)<5 u_{3}(y)-u_{3}(b) \quad \text { and } \quad 3 u_{4}(x)+u_{4}(b)<5 u_{4}(y)-u_{4}(a) .
$$

Note that the veto players both prefer $y$ to $x$, but no other player agrees, and thus it is not the case that $y \succ x$. It is readily checked that if the discount factor $\delta$ is sufficiently close to one, then there is a stationary bargaining equilibrium that generates the Markov chain depicted in Figure $2,{ }^{7}$ where arrows represent transitions, labeled by the transition probabilities and the coalitions that support the transitions (the proposers who initiate the transitions are starred). Moreover, this equilibrium is such that: given status quo $x$, each player proposes her favorite alternative in $\{y, a, b\}$, and this proposal passes; given status quo $y$, all players maintain the status quo; given status quo $a$, player 3 maintains the status quo, whereas all other players obtain the outcome $y$; with analogous actions at status quo $b$. In particular, given status quo $x$, players 3 and 4 are willing to vote for proposal $y$ in order to avoid obtaining their least preferred alternative, which occurs with probability $1 / 4$ if the status quo is maintained - our assumptions guarantee that, for $\delta$ sufficiently close to one, $V_{i}(x \mid \sigma)<V_{i}(y \mid \sigma)$ for each $i=3$, 4. And given status quo $x$, players 1 and 2 are willing to vote for any of $a$ and $b$ in order to avoid remaining at their least preferred alternative for another period. Here, the set of absorbing alternatives is $A(\sigma)=\{y\}$, and thus we have $x \notin A(\sigma)$, yet it is not the case that $y \succ x$, violating external stability.

The next result strengthens the assumptions of Theorem 2 to provide a tight connection between equilibrium ergodic sets and vNM solutions: if the voting rule is oligarchical, then for every stationary bargaining equilibrium, the set of absorbing points is equal to the unique vNM solution.

Theorem 3: Assume $\mathcal{D}$ is oligarchical, and that there is at least one veto player with positive recognition probability, i.e., there exists $i \in \bigcap \mathcal{D}$ with $\rho_{i}>0$. Then there exists $\bar{\delta} \in(0,1)$ such that for all $\delta \in(\bar{\delta}, 1)$ and all stationary bargaining equilibria $\sigma, A(\sigma)$ is the unique von Neumann-Morgenstern solution.

\footnotetext{
${ }^{7}$ Details are provided in the supplementary appendix.
} 


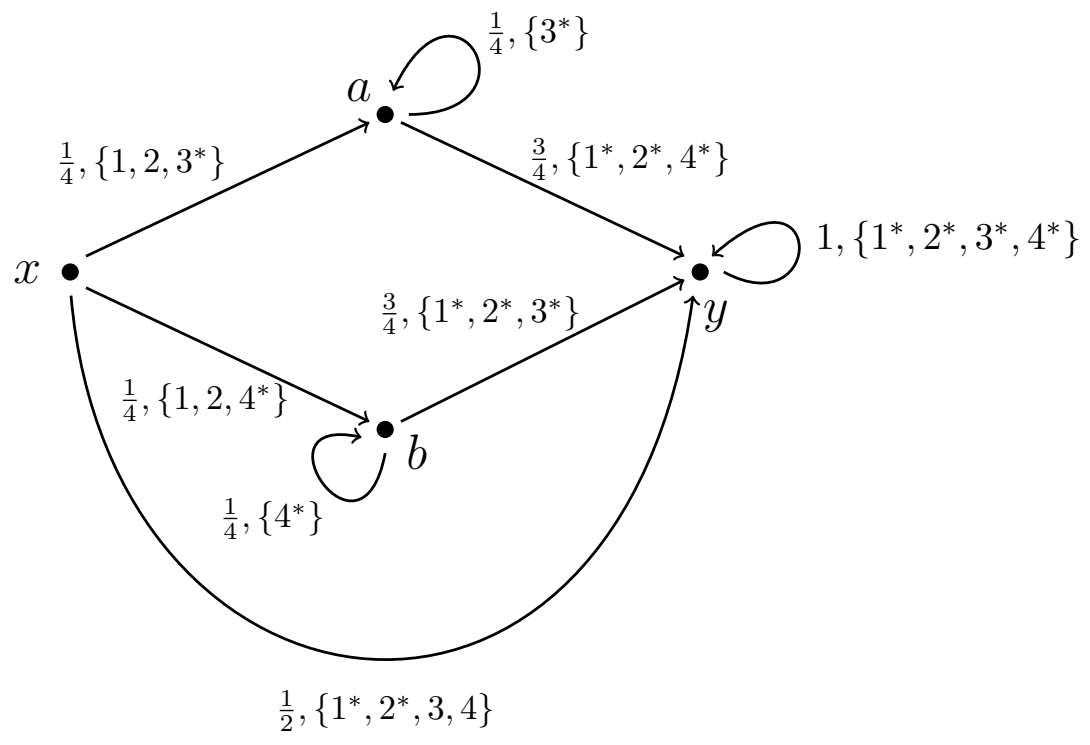

Figure 2: Multiple paths

Proof: One direction follows from Anesi (2010). For the other, consider any sequence $\left\{\delta^{k}\right\}$ of discount factors converging to one and any corresponding sequence $\left\{\sigma^{k}\right\}$ of stationary bargaining equilibria. We must show that for high enough $k$, the set $A\left(\sigma^{k}\right)$ of absorbing points of $\sigma^{k}$ is equal to the vNM solution. Let player $i$ be a vetoer with $\rho_{i}>0$. Clearly, the set $A\left(\sigma^{k}\right)$ is internally stable, for else there exist $x, y \in A\left(\sigma^{k}\right)$ such that $x \succ y$, but then given status quo $y$, player $i$ could successfully propose $x$, contradicting the fact that $y$ is an absorbing alternative. To establish external stability, consider any $x_{k} \in X \backslash A\left(\sigma^{k}\right)$. By Theorem 2, for sufficiently high $k$, there exists $y_{k}$ such that $A\left(x_{k} \mid \sigma^{k}\right)=\left\{y_{k}\right\}$, so that starting from $x_{k}$, the equilibrium Markov process is absorbed into $y_{k}$. Letting $\left\{\Gamma\left(\sigma^{k}\right)\right\}$ be the corresponding sequence of equilibrium graphs, we can also identify an alternative $w_{k}$ that minimizes player $i$ 's dynamic payoff over the outcomes distinct from $x_{k}$ that occur with positive probability given status quo $x_{k}$, i.e.,

$$
V_{i}\left(w_{k} \mid \sigma^{k}\right)=\min _{z \neq x_{k}: x_{k} \Gamma\left(\sigma^{k}\right) z} V_{i}\left(z \mid \sigma^{k}\right) .
$$

Since $X$ is finite, we can go to a subsequence (still indexed by $k$ ) on which 
these alternatives and graphs are constant, and henceforth we write $x=x_{k}$, $y=y_{k}, w=w_{k}$, and $\Gamma=\Gamma\left(\sigma^{k}\right)$. Let $\left\{z_{1}, \ldots, z_{m}\right\}$ be a path from $w$ to $y$, so that

$$
w=z_{1} \Gamma z_{2} \cdots \Gamma z_{m-1} \Gamma z_{m}=y .
$$

Since $i$ is a veto player, it follows that $V_{i}\left(w \mid \sigma^{k}\right) \geq V_{i}\left(x \mid \sigma^{k}\right)$ and that for each $h=1, \ldots, m-1$, we have $V_{i}\left(z_{h+1} \mid \sigma^{k}\right) \geq V_{i}\left(z_{h} \mid \sigma^{k}\right)$. In particular, we have $u_{i}(y) \geq V_{i}\left(w \mid \sigma^{k}\right)$. Now suppose toward a contradiction that we have $u_{i}(x)>V_{i}\left(w \mid \sigma^{k}\right)$. Then

$$
\begin{aligned}
& V_{i}\left(x \mid \sigma^{k}\right) \\
& \quad=(1-\delta) u_{i}(x)+\delta P\left(x, x \mid \sigma^{k}\right) V_{i}\left(x \mid \sigma^{k}\right)+\delta \sum_{z \neq x} P\left(x, z \mid \sigma^{k}\right) V_{i}\left(z \mid \sigma^{k}\right) \\
& \quad>(1-\delta) V_{i}\left(w \mid \sigma^{k}\right)+\delta P\left(x, x \mid \sigma^{k}\right) V_{i}\left(x \mid \sigma^{k}\right)+\delta\left[1-P\left(x, x \mid \sigma^{k}\right)\right] V_{i}\left(w \mid \sigma^{k}\right),
\end{aligned}
$$

which implies

$$
V_{i}\left(x \mid \sigma^{k}\right)>V_{i}\left(w \mid \sigma^{k}\right),
$$

a contradiction. We conclude that $u_{i}(y) \geq V_{i}\left(w \mid \sigma^{k}\right) \geq u_{i}(x)$, which further implies $u_{i}(y)>u_{i}(x)$. This inequality holds for every veto player, and since the voting rule is oligarchical, we conclude that $y \succ x$, as required. Q.E.D.

The following example illustrates Theorem 3 with the variation of the game in Example 3, modified so as to have an oligarchical voting rule.

Example 4: In Example 3, we examined a game in which the voting rule was collegial, but not oligarchical, and we constructed a stationary bargaining equilibrium (see Figure 2) whose absorbing set was not a vNM solution. Consider now the same game but with oligarchical voting rule $\mathcal{D}=\{C \mid 1,2 \in C\}$, so that players 1 and 2 no longer need an additional vote for passage of a proposal. In this case, $\{y\}$ is the unique vNM solution. It is easy to see that the strategy profile depicted in Figure 2 is still a stationary bargaining equilibrium: ${ }^{8}$ all successful proposals to amend status quos are accepted by players 1 and 2, and the latter successfully propose their ideal alternative $y$ (which is absorbing) at any status quo. But the absorbing set $\{y\}$ of this equilibrium now coincides with the unique vNM solution for the oligarchical rule $\mathcal{D}$. Moreover, it follows from Theorem 3 that, in this game, there cannot be a stationary bargaining equilibrium with an absorbing set that differs from $\{y\}$.

\footnotetext{
${ }^{8}$ Recall that arrows represent transitions, labeled by the transition probabilities and the coalitions that support the transitions, and that the proposers who initiate the transitions are starred.
} 


\section{Persistent Agenda Setter}

It is worthwhile to summarize briefly the steps in the analysis above. First, Theorem 1 assumes that the Nakamura number of the voting rule is high relative to the number of alternatives; then Theorem 2 adds more structure by assuming a veto player. The additional structure of an oligarchic rule is then used in Theorem 3 to obtain a characterization of stationary bargaining equilibria in terms of vNM solutions. Our next theorem adds structure to Theorems 1 and 2 in a different direction: it casts the analysis into the persistent agenda setter model (Diermeier and Fong 2011, 2012), in which some player $i$ is given the sole power to make proposals.

Let $\mathcal{D}^{i}$ be the voting rule obtained from $\mathcal{D}$ by adding player $i$ to every decisive coalition in $\mathcal{D}$, that is, $\mathcal{D}^{i}=\{C \cup\{i\}: C \in \mathcal{D}\}$. If player $i$ is the single agenda setter (i.e., $\rho_{i}=1$ ), then any stationary bargaining equilibrium $\sigma$ with rule $\mathcal{D}$ is also a stationary bargaining equilibrium with rule $\mathcal{D}^{i}$. To see this, note that if $\sigma$ is not an equilibrium with rule $\mathcal{D}^{i}$ then, at some status quo $x, i$ must propose an alternative $y$ that is accepted with rule $\mathcal{D}$ and rejected with rule $\mathcal{D}^{i}$. This implies that $i$ rejects this proposal and, therefore, that (with rule $\mathcal{D}$ ) she would have been strictly better off maintaining status quo $x$ rather than proposing $y$; a contradiction. Hence, Theorems 1 and 2 can be applied directly to any voting rule $\mathcal{D}$ in the persistent agenda setter model: their conclusions apply to all stationary bargaining equilibria with collegial rule $\mathcal{D}^{i}$, including all those that are also stationary bargaining equilibria with rule $\mathcal{D}$. This yields the following corollary.

Corollary 1: Assume that there is a persistent agenda setter, i.e., there exists $i \in\{1, \ldots, n\}$ with $\rho_{i}=1$. Then there exists $\bar{\delta} \in(0,1)$ such that for all $\delta \in(\bar{\delta}, 1)$, all stationary bargaining equilibria $\sigma$, and all $x \in X$, $|A(x \mid \sigma)|=1$.

The preceding argument does not directly yield a version of Theorem 3 for the persistent agenda setter model, as the theorem assumes an oligarchic rule. Nevertheless, the structure of a single proposer allows us to obtain a sharper result than Theorem 2, which we will draw on to obtain a characterization of vNM solutions. Our final theorem indeed establishes that this structure, in addition to ensuring the uniqueness of the absorbing alternative $y$ from any status quo $x$, implies that there is a unique path determined in equilibrium from $x$ to $y$. In particular, the equilibrium graph $\Gamma(\sigma)$ possesses no "branches," so that for every alternative $x$, there is a unique alterna- 
tive $z$ such that $x \Gamma(\sigma) z$, precluding equilibrium Markov chains of the sort demonstrated in Example 3. Note the further implication that under the conditions of the theorem, stationary bargaining equilibria are essentially pure, in the sense that for every non-absorbing alternative $x \notin A(\sigma)$, the proposal strategy $\pi_{i}(x, \cdot)$ puts probability one on the single alternative $z$ such that $x \Gamma(\sigma) z$; if $x$ is an absorbing alternative, then the agenda setter may mix between proposals that are rejected, but mixing in this case is nominal. Thus, we find that Diermeier and Fong's (2012) restriction to pure strategy equilibria is redundant.

Theorem 4: Assume that there is a persistent agenda setter, i.e., there exists $i \in\{1, \ldots, n\}$ with $\rho_{i}=1$. Then there exists $\bar{\delta} \in(0,1)$ such that for all $\delta \in(\bar{\delta}, 1)$, all stationary bargaining equilibria $\sigma$, and all $x \in X$, there exist a unique absorbing alternative $y \in X$, a unique natural number $m \geq 1$, and unique alternatives $z_{1}, \ldots, z_{m} \in X$ (which may depend on $\sigma$ and $x$ ) such that

$$
x \Gamma(\sigma) z_{1} \Gamma(\sigma) \cdots z_{m-1} \Gamma(\sigma) z_{m}=y .
$$

Proof: Consider an arbitrary sequence $\left\{\delta^{k}\right\}$ of discount factors converging to one and a corresponding sequence $\left\{\sigma^{k}\right\}$ of stationary bargaining equilibria. Going to a subsequence (still indexed by $k$ ), we can assume that the corresponding graph, $\Gamma$ is constant. Consider an alternative $x$. By Corollary 1 , there exists a unique alternative $y$ such that $A\left(x \mid \sigma^{k}\right)=\{y\}$ for all $k \in \mathbb{N}$. It suffices to show that for sufficiently high $k$, there is a unique path between $x$ and $y$. We proceed in three steps:

Step 1: The alternative y maximizes player i's stage utility over the set $R(x)$ of alternatives reachable from $x$. Although $i$ may not be a veto player, being the single agenda setter, she can unilaterally maintain the status quo. Let $b$ maximize player $i$ 's stage utility over the alternatives reachable from $x$, i.e., $u_{i}(b)=\max _{z \in R(x)} u_{i}(z)$. Since $R(b) \subseteq R(x)$, it follows that $u_{i}(b) \geq \max _{z \in R(b)} u_{i}(z)$ and thus $V_{i}\left(b \mid \sigma^{k}\right) \geq u_{i}(b)>V_{i}\left(z \mid \sigma^{k}\right)$ for all $z \in R(b) \backslash\{b\}$. Since player $i$ 's proposal strategy is optimal, we conclude that $b$ is absorbing, and since $y$ is the unique absorbing alternative reachable from $x$, we have $y=b$.

Step 2: $\Gamma$ is acyclic on $R(x)$. Suppose toward a contradiction that there exist $x_{1}, \ldots, x_{m}$ in $R(x)$ such that $x_{1} \Gamma x_{2} \Gamma \cdots x_{m} \Gamma x_{1}$. This implies that

$$
V_{i}\left(x_{1} \mid \sigma^{k}\right)=\cdots=V_{i}\left(x_{m} \mid \sigma^{k}\right) .
$$


Note that the agenda setter $i$ solves a dynamic programming problem, and $\sigma_{i}^{k}$ is optimal, given $\sigma_{-i}^{k}$. Since $x_{2}$ is proposed with positive probability given status quo $x_{1}$, it is an optimal choice at $x_{1}$, given that future choices are made according to $\sigma_{i}^{k}$. Then it is optimal to always choose $x_{2}$ at $x_{1}$, using $\sigma_{i}^{k}$ at all other status quos. Call this strategy $\sigma_{i}^{k, 1}$. We then modify $\sigma_{i}^{k, 1}$ so that at $x_{2}$, the agenda setter chooses $x_{3}$ with probability one. The resulting strategy, $\sigma_{i}^{k, 2}$, is also optimal. In general, we modify $\sigma_{i}^{k, j}$ so that at status quo $x_{j}$, the agenda setter chooses $x_{j+1}$ with probability one, giving us an optimal strategy at each step. In the end, the strategy $\sigma_{i}^{k, m}$ is optimal, but following it, the agenda setter just cycles through $x_{1}, \ldots, x_{m}$. It follows from Step 1 that the agenda setter's payoff from $\sigma_{i}^{k, m}$, starting from $x_{1}$, is bounded above by

$$
\max \left\{u_{i}\left(x_{j}\right) \mid j=1, \ldots, m\right\}<u_{i}(y) .
$$

For sufficiently high $k$, this payoff is less than the payoff from following $\sigma_{i}^{k}$, because following that strategy $y$ is eventually reached with probability one. This contradiction completes the step.

Step 3. There is a unique path between $x$ and $y$ in $\Gamma$. Note that acyclicity of $\Gamma$ implies irreflexivity, so that $x \notin R(x)$. Thus, it follows from Step 2 that if $z \in R(x)$, then $z \neq x$ and $R(z) \varsubsetneqq R(x)$. Say $z$ branches if there are distinct alternatives $s$ and $t$ such that $z \Gamma s$ and $z \Gamma t$. Suppose toward a contradiction that some alternative $z \in R(x)$ branches, and choose $z$ so that $R(z)$ is minimal among

$$
\{R(w) \mid w \in R(x) \text { and } w \text { branches }\}
$$

according to set inclusion. Note that no alternatives reachable from $z$ can branch. Then following $z$ are at least two deterministic paths that lead to $y$. But as $\delta^{k} \rightarrow 1$, it is not possible to maintain the agenda setter's indifference over these paths for arbitrarily high $k$.

Q.E.D.

Next, we record an implication of Theorem 4 for the connections between vNM solutions and equilibrium absorbing sets in the persistent agenda setter model. Note that this corollary imposes no restriction on the voting rule $\mathcal{D}$. The result thus extends Theorem 1 in Diermeier and Fong (2012) by generalizing their quota rules to an arbitrary voting rule and by removing the restriction to pure strategy equilibria.

Corollary 2: Assume that there is a persistent agenda setter, i.e., there exists $i \in\{1, \ldots, n\}$ with $\rho_{i}=1$. Then there exists $\bar{\delta} \in(0,1)$ such that for all 
$\delta \in(\bar{\delta}, 1)$ and all subsets $Y \subseteq X$, there is a stationary bargaining equilibrium $\sigma$ with $A(\sigma)=Y$ if and only if $Y$ is a von Neumann-Morgenstern solution for $\mathcal{D}^{i}$.

Proof: Sufficiency follows from Diermeier and Fong (2012). ${ }^{9}$ For necessity, suppose toward a contradiction that there are a sequence $\left\{\delta^{k}\right\}$ of discount factors converging to one and a sequence $\left\{\sigma^{k}\right\}$ of stationary bargaining equilibria such that for each $k$, the set $A\left(\sigma^{k}\right)$ of absorbing points of $\sigma^{k}$ is not a vNM solution. By the same logic as in the proof of Theorem 3, the set $A\left(\sigma^{k}\right)$ must be internally stable. It follows that $A\left(\sigma^{k}\right)$ violates external stability, so there exists $x^{k} \in X \backslash A\left(\sigma^{k}\right)$ such that for all $y \in A\left(\sigma^{k}\right)$, it is not the case that $y \succ x^{k}$. By Corollary 1 and Theorem 4, for sufficiently high $k$, there exist: an alternative $y^{k}$ such that $A\left(x^{k} \mid \sigma^{k}\right)=\left\{y^{k}\right\}$; a unique path $\left\{x^{k}, z_{1}^{k}, \ldots, z_{m_{k}}^{k}\right\}$ from $x^{k}$ to $y^{k}$; and a coalition $C^{k} \in \mathcal{D}^{i}$ such that $V_{j}\left(x^{k} \mid \sigma^{k}\right) \leq V_{j}\left(z_{1}^{k} \mid \sigma^{k}\right)$ for all $j \in C^{k}$. Going to a subsequence (still indexed by $k$ ), we can assume that this coalition and these alternatives are constant, and henceforth we write $C=C^{k}, x=x^{k}, y=y^{k}$, and $z_{1}=z_{1}^{k}$. Hence, for each $k$, we have

$$
\begin{aligned}
0 & \leq V_{j}\left(z_{1} \mid \sigma^{k}\right)-V_{j}\left(x \mid \sigma^{k}\right) \\
& =V_{j}\left(z_{1} \mid \sigma^{k}\right)-\left(1-\delta^{k}\right) u_{j}(x)-\delta^{k} V_{j}\left(z_{1} \mid \sigma^{k}\right) \\
& =\left(1-\delta^{k}\right)\left(V_{j}\left(z_{1} \mid \sigma^{k}\right)-u_{j}(x)\right)
\end{aligned}
$$

for all $j \in C$. This implies $V_{j}\left(z_{1} \mid \sigma^{k}\right) \geq u_{j}(x)$ for all $j \in C$. Taking limits and using $V_{j}\left(z_{1} \mid \sigma^{k}\right) \rightarrow u_{j}(y)$, we then have $u_{j}(y) \geq u_{j}(x)$ and, thus, $u_{j}(y)>u_{j}(x)$ for all $j \in C$. This contradicts our supposition that there is no absorbing alternative $y$ such that $y \succ x$. Q.E.D.

To illustrate Corollary 2, we provide an example with three alternatives in which starting from any given status quo, equilibrium outcomes are eventually absorbed to the ideal point of the agenda setter. Of note, the example shows that equilibrium dynamics can be non-trivial: even though all players can anticipate the final outcome, this is not achieved immediately; rather, starting from the worst alternative of the agenda setter, the agenda setter's ideal point is obtained incrementally, first visiting the middle-ranked alternative of the agenda setter, and then the ideal alternative. In other words, the equilibrium features delayed gratification, as the agenda setter exploits a wedge between the preferences of the other players to achieve her ideal point in no more than two steps.

\footnotetext{
${ }^{9}$ Their equilibrium construction does not rely on the assumption that $\mathcal{D}$ is a quota rule.
} 


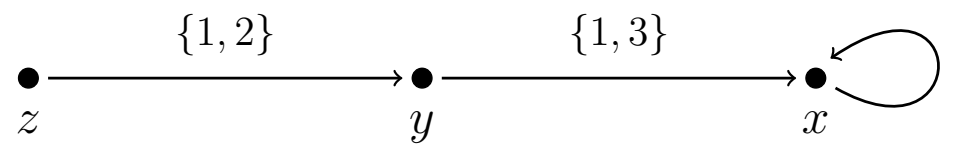

Figure 3: Delayed Gratification for the Agenda Setter

Example 5: Let the set of alternatives be $X=\{x, y, z\}$, let there be three players, with player 1 the agenda setter. Assume that the voting rule is such that $\mathcal{D}^{1}=\{\{1,2\},\{1,3\},\{1,2,3\}\}$, i.e., the agenda setter needs the support of at least one other player to pass a proposal. Stage payoffs satisfy

$$
u_{1}(x)>u_{1}(y)>u_{1}(z), u_{2}(y)>u_{2}(x)>u_{2}(z) \text {, and } u_{3}(z)>u_{3}(x)>u_{3}(y) .
$$

Thus, the unique vNM solution for $\mathcal{D}^{1}$ is the singleton $\{x\}$, and Corollary 2 implies that when players are sufficiently patient, $x$ is the unique equilibrium absorbing point. To investigate dynamics in more detail, assume that

$$
u_{i}(x)<\frac{u_{i}(y)+u_{i}(z)}{2}
$$

for players $i=2,3$. It can be shown that when $\delta$ is sufficiently close to one, ${ }^{10}$ there is a stationary bargaining equilibrium that generates the Markov chain depicted in Figure 3, where arrows represent transitions and are labeled by the coalitions that support the transitions. Note that beginning from status quo $z$, the outcome is eventually absorbed into $x$, the ideal point of the agenda setter, but this process takes two periods. Even though all players know that $x$ will eventually be reached, the agenda setter must exploit a wedge between the preferences of the other players, leveraging player 2's strong preference for $y$ (relative to $x$ ) to compromise player 3 , who then assents to the move from status quo $y$ to alternative $x$. It is worth noting that there also exists a stationary bargaining equilibrium in which the agenda setter successfully proposes $x$ from any status quo - the construction is similar to Diermeier and Fong's (2011). This shows that there can be multiple equilibria, even when the vNM solution is unique, and that dynamics can depend on the equilibrium.

\footnotetext{
${ }^{10}$ Details are provided in the supplementary appendix.
} 


\section{References}

V. Anesi (2010) "Noncooperative Foundations of Stable Sets in Voting Games," Games and Economic Behavior 70, 488-493.

V. ANESI (2012) "A New Old Solution for Weak Tournaments," Social Choice and Welfare 39, 919-930.

V. Anesi And D.J. Seidmann (2014) "Bargaining over an Endogenous Agenda," Theoretical Economics 9, 445-482.

V. Anesi And D.J. Seidmann (2015) "Bargaining in Standing Committees with an Endogenous Default," Review of Economic Studies 82, $825-867$.

D.P. BAron (1996) "A Dynamic Theory of Collective Goods Programs," American Political Science Review 90, 316-330.

D.P. Baron And T.R. Bowen (2014) "Dynamic Coalitions," working paper.

M. Battaglini and T. Palfrey (2012) "The Dynamics of Distributive Politics," Economic Theory 49, 739-777.

T.R. Bowen and Z. Zahran (2012) "On Dynamic Compromise," Games and Economic Behavior 76, 391-419.

D. Diermeier, G. Egorov and K. Sonin (2013) "Endogenous Property Rights," National Bureau of Economic Research Working paper Series No. 19734.

D. Diermeier And P. Fong (2011) "Legislative Bargaining with Reconsideration," Quarterly Journal of Economics 126, 947-985.

D. Diermeier And P. Fong (2012) "Characterization of the von NeumannMorgenstern Stable Set in a Non-Cooperative Model of Dynamic PolicyMaking with a Persistent Agenda Setter," Games and Economic Behavior 76, 349-353.

J. Duggan and T. Kalandrakis (2012) "Dynamic Legislative Policy Making," Journal of Economic Theory 147, 1653-1688.

W. Dziuda And A. Loeper (2015) "Dynamic Collective Choice with Endogenous Status Quo," Journal of Political Economy, forthcoming. 
A. Kalandrakis (2004) "A Three-Player Dynamic Majoritarian Bargaining Game," Journal of Economic Theory 116, 294-322.

A. Kalandrakis (2010) "Minimum Winning Coalitions and Endogenous Status Quo," International Journal of Game Theory, 39, 617-643.

R.D. McKelvey, P.C. Ordeshook and M.D. Winer (1978) "The Competitive Solution for $N$-Person Games without Transferable Utility, with an Application to Committee Games." American Political Science Review 72, 599-615.

S. Muto (1984) "Stable Sets for Simple Games with Ordinal Preferences," Journal of Operations Research Society of Japan 27, 250-259.

S. Nunnari (2014) "Dynamic Legislative Bargaining with Veto Power: Theory and Experiments," working paper.

M. Richter (2014) "Fully Absorbing Dynamic Compromise," Journal of Economic Theory 152, 92-104.

J. von Neumann and O. Morgenstern (1944). Theory of Games and Economic Behavior. Princeton University Press, Princeton.

J. ZÁpAl (2014) "Simple Markovian Equilibria in Dynamic Spatial Legislative Bargaining," working paper. 
Supplementary Appendix (Not for Publication):
Details of the Equilibria in Examples 2,3 And 5

Example 2. Let the strategy profile $\sigma$ be defined by:

$$
\begin{aligned}
& \pi_{1}(x, x)=\pi_{1}(y, z)=\pi_{1}(z, x)=\pi_{2}(x, y)=\pi_{2}(y, z)=\pi_{2}(z, z) \\
& =\pi_{3}(x, y)=\pi_{3}(y, y)=\pi_{3}(z, x)=1 \\
& \alpha_{2}(x, y)=\alpha_{3}(x, y)=1-\alpha_{1}(x, y)=1 ; \alpha_{1}(x, z)=\alpha_{3}(x, z)=1-\alpha_{2}(x, z)=0 ; \\
& \alpha_{2}(y, x)=\alpha_{3}(y, x)=1-\alpha_{1}(y, x)=0 ; \alpha_{1}(y, z)=\alpha_{2}(y, z)=1-\alpha_{3}(y, z)=1 ; \\
& \alpha_{1}(z, x)=\alpha_{3}(z, x)=1-\alpha_{2}(z, x)=1 ; \alpha_{1}(z, y)=\alpha_{2}(z, y)=1-\alpha_{3}(z, y)=0 .
\end{aligned}
$$

Hence,

$$
\begin{aligned}
& \alpha(x, x)=\alpha(x, y)=1-\alpha(x, z)=1 \\
& \alpha(y, y)=\alpha(y, z)=1-\alpha(y, x)=1 \\
& \alpha(z, z)=\alpha(z, x)=1-\alpha(z, y)=1 .
\end{aligned}
$$

Simple calculations then yield

$$
\begin{aligned}
& V_{i}(x \mid \sigma)=\frac{(3-\delta)^{2} u_{i}(x)+2(3-\delta) \delta u_{i}(y)+4 \delta^{2} u_{i}(z)}{3\left(3+\delta^{2}\right)}, \\
& V_{i}(y \mid \sigma)=\frac{4 \delta^{2} u_{i}(x)+(3-\delta)^{2} u_{i}(y)+2(3-\delta) \delta u_{i}(z)}{3\left(3+\delta^{2}\right)} \\
& V_{i}(z \mid \sigma)=\frac{2(3-\delta) \delta u_{i}(x)+4 \delta^{2} u_{i}(y)+(3-\delta)^{2} u_{i}(z)}{3\left(3+\delta^{2}\right)}
\end{aligned}
$$

so that

$$
\begin{aligned}
& V_{i}(x \mid \sigma) \geq V_{i}(y \mid \sigma) \text { iff }(3+\delta) u_{i}(x) \geq(3-\delta) u_{i}(y)+2 \delta u_{i}(z) \\
& V_{i}(x \mid \sigma) \geq V_{i}(z \mid \sigma) \text { iff }(3-\delta) u_{i}(x)+2 \delta u_{i}(y) \geq(3+\delta) u_{i}(z), \text { and } \\
& V_{i}(y \mid \sigma) \geq V_{i}(z \mid \sigma) \text { iff }(3+\delta) u_{i}(y) \geq 2 \delta u_{i}(x)+(3-\delta) u_{i}(z)
\end{aligned}
$$

for each $i=1,2,3$. Thus, under our assumptions on stage utilities, dynamic payoffs satisfy $V_{1}(y \mid \sigma)<V_{1}(z \mid \sigma)<V_{1}(x \mid \sigma), V_{2}(x \mid \sigma)<V_{2}(y \mid \sigma)<V_{2}(z \mid \sigma)$, and $V_{3}(z \mid \sigma)<V_{3}(x \mid \sigma)<V_{3}(y \mid \sigma)$. Combined with equations (3)-(5), these inequalities imply that $\sigma$ satisfies the conditions for a stationary bargaining equilibrium. 
Example 3. The strategy profile $\sigma$, described in the example, is defined by

- Policy strategies given status quo $x: \pi_{1}(x, y)=\pi_{2}(x, y)=\pi_{3}(x, a)=$ $\pi_{4}(x, b)=1$

- Policy strategies given status quo $y: \pi_{i}(y, y)=1$ for each $i=1,2,3,4$;

- Policy strategies given status quo $a: \pi_{1}(a, y)=\pi_{2}(a, y)=\pi_{3}(a, a)=$ $\pi_{4}(a, y)=1$

- Policy strategies given status quo $b: \pi_{1}(b, y)=\pi_{2}(b, y)=\pi_{3}(b, y)=$ $\pi_{4}(b, b)=1$

- Voting strategies given status quo $x: \alpha_{i}(x, y)=1$ for each $i=1,2,3,4$,

$$
\begin{aligned}
& \alpha_{1}(x, a)=\alpha_{2}(x, a)=\alpha_{3}(x, a)=1-\alpha_{4}(x, a)=1, \\
& \alpha_{1}(x, b)=\alpha_{2}(x, b)=\alpha_{4}(x, b)=1-\alpha_{3}(x, b)=1,
\end{aligned}
$$

so that

$$
\alpha(x, x)=\alpha(x, y)=\alpha(x, a)=\alpha(x, b)=1 ;
$$

- Voting strategies given status quo $y: \alpha_{i}(y, x)=0$ for each $i=1,2,3,4$,

$$
\begin{aligned}
& \alpha_{1}(y, a)=\alpha_{2}(y, a)=\alpha_{4}(y, a)=1-\alpha_{3}(y, a)=0, \\
& \alpha_{1}(y, b)=\alpha_{2}(y, b)=\alpha_{3}(y, b)=1-\alpha_{4}(y, b)=0,
\end{aligned}
$$

so that

$$
\alpha(y, x)=1-\alpha(y, y)=\alpha(y, a)=\alpha(y, b)=0 ;
$$

- Voting strategies given status quo $a$ :

$$
\begin{aligned}
& \alpha_{1}(a, x)=\alpha_{2}(a, x)=\alpha_{3}(a, x)=1-\alpha_{4}(a, x)=0, \\
& \alpha_{1}(a, y)=\alpha_{2}(a, y)=\alpha_{4}(a, y)=1-\alpha_{3}(a, y)=1, \\
& \alpha_{1}(a, b)=1-\alpha_{2}(a, b)=\alpha_{3}(a, b)=1-\alpha_{4}(a, b)=0
\end{aligned}
$$

so that

$$
1-\alpha(a, x)=\alpha(a, y)=\alpha(a, a)=1-\alpha(a, b)=1 ;
$$


- Voting strategies given status quo $b$ :

$$
\begin{aligned}
& \alpha_{1}(b, x)=\alpha_{2}(b, x)=\alpha_{4}(b, x)=1-\alpha_{3}(b, x)=0, \\
& \alpha_{1}(b, y)=\alpha_{2}(b, y)=\alpha_{3}(b, y)=1-\alpha_{4}(b, y)=1, \\
& \alpha_{1}(b, a)=1-\alpha_{2}(b, a)=\alpha_{3}(b, a)=1-\alpha_{4}(b, a)=1, \\
& 1-\alpha(b, x)=\alpha(b, y)=1-\alpha(b, a)=\alpha(b, b)=1 .
\end{aligned}
$$

It is readily checked that the corresponding dynamic payoffs are:

$$
\begin{aligned}
& V_{i}(y \mid \sigma)=u_{i}(y) \\
& V_{i}(a \mid \sigma)=u_{i}(a)+\frac{3 \delta}{4-\delta}\left[u_{i}(y)-u_{i}(a)\right] \\
& V_{i}(b \mid \sigma)=u_{i}(b)+\frac{4(1-\delta)}{4-\delta}\left[u_{i}(b)-u_{i}(y)\right] \\
& V_{i}(x \mid \sigma)=(1-\delta) u_{i}(x)+\frac{\delta}{4}\left[2 V_{i}(y \mid \sigma)+V_{i}(a \mid \sigma)+V_{i}(b \mid \sigma)\right]
\end{aligned}
$$

for each $i=1,2,3,4$. Furthermore, we have

$$
\frac{V_{i}(a \mid \sigma)-V_{i}(x \mid \sigma)}{1-\delta}=u_{i}(a)-u_{i}(x)+\frac{\delta}{4-\delta}\left[u_{i}(y)-u_{i}(b)\right]
$$

and

$$
\frac{V_{i}(b \mid \sigma)-V_{i}(x \mid \sigma)}{1-\delta}=u_{i}(b)-u_{i}(x)+\frac{\delta}{4-\delta}\left[u_{i}(y)-u_{i}(a)\right],
$$

for every $i=1,2,3,4$. From our assumptions on $u_{1}$ and $u_{2}$, it follows that

$$
V_{1}(x \mid \sigma)<V_{1}(b \mid \sigma)<V_{1}(a \mid \sigma)<V_{1}(y \mid \sigma)
$$

and

$$
V_{2}(x \mid \sigma)<V_{2}(a \mid \sigma)<V_{2}(b \mid \sigma)<V_{2}(y \mid \sigma) .
$$

In addition,

$$
\frac{V_{i}(y \mid \sigma)-V_{i}(x \mid \sigma)}{1-\delta}=u_{i}(y)-u_{i}(x)+\frac{\delta}{4-\delta}\left[2 u_{i}(y)-u_{i}(a)-u_{i}(b)\right]
$$

for each $i=1,2,3,4$. Combined with these equalities, our assumptions on $u_{3}$ and $u_{4}$ imply that there exist $\bar{\delta}_{3}, \bar{\delta}_{4} \in(0,1)$ such that, for each $i=3,4$, $V_{i}(y \mid \sigma)>V_{i}(x \mid \sigma)$ for all $\delta \in\left(\bar{\delta}_{i}, 1\right)$; so that

$$
V_{3}(b \mid \sigma)<V_{3}(x \mid \sigma)<V_{3}(y \mid \sigma)<V_{3}(a \mid \sigma)
$$


whenever $\delta>\bar{\delta}_{3}$, and

$$
V_{4}(a \mid \sigma)<V_{4}(x \mid \sigma)<V_{4}(y \mid \sigma)<V_{4}(b \mid \sigma)
$$

whenever $\delta>\bar{\delta}_{4}$.

Suppose $\delta>\max \left\{\bar{\delta}_{3}, \bar{\delta}_{4}\right\}$. Inequalities (10)-(13) imply that the voting strategies defined above satisfy condition (ii) in the definition of a stationary bargaining equilibrium. Coupled with (6)-(9), these inequalities also imply that the proposal strategies defined above satisfy condition (i) in that definition. This proves that $\sigma$ is a stationary bargaining equilibrium.

Example 5. The strategy profile $\sigma$, described in the example, is defined by

- Policy strategy given status quo $x: \pi_{1}(x, x)=1$;

- Policy strategy given status quo $y: \pi_{1}(y, x)=1$;

- Policy strategy given status quo $z: \pi_{1}(z, y)=1$;

- Voting strategies given status quo $x$ :

$$
\begin{aligned}
& 1-\alpha_{1}(x, y)=\alpha_{2}(x, y)=1-\alpha_{3}(x, y)=1 \\
& 1-\alpha_{1}(x, z)=\alpha_{2}(x, z)=\alpha_{3}(x, z)=1
\end{aligned}
$$

so that the agenda setter can maintain status quo $x$, of course, or successfully propose $z$ (but not $y$ ), if desired;

- Voting strategies given status quo $y$ :

$$
\begin{aligned}
\alpha_{1}(y, x) & =1-\alpha_{2}(y, x)=\alpha_{3}(y, x)=1, \\
1-\alpha_{1}(y, z) & =1-\alpha_{2}(y, z)=\alpha_{3}(y, z)=1,
\end{aligned}
$$

so that the agenda setter could maintain status quo $y$ or successfully propose $x$ (but not $z$ ), if desired;

- Voting strategies given status quo $z$ :

$$
\begin{aligned}
& \alpha_{1}(z, x)=1-\alpha_{2}(z, x)=1-\alpha_{3}(z, x)=1 \\
& \alpha_{1}(z, y)=\alpha_{2}(z, y)=1-\alpha_{3}(z, y)=1
\end{aligned}
$$

so that the agenda setter could maintain status quo $z$ or successfully propose alternative $y$ (but not $x$ ). 
It is readily checked that the corresponding dynamic payoffs are:

$$
\begin{aligned}
& V_{i}(x \mid \sigma)=u_{i}(x) \\
& V_{i}(y \mid \sigma)=(1-\delta) u_{i}(y)+\delta u_{i}(x) \\
& V_{i}(z \mid \sigma)=(1-\delta)\left(u_{i}(z)+\delta u_{i}(y)\right)+\delta^{2} u_{i}(x)
\end{aligned}
$$

for each $i=1,2,3$. Furthermore, we have

$$
\begin{aligned}
& \frac{V_{i}(x \mid \sigma)-V_{i}(y \mid \sigma)}{1-\delta}=u_{i}(x)-u_{i}(y) \\
& \frac{V_{i}(x \mid \sigma)-V_{i}(z \mid \sigma)}{1-\delta}=u_{i}(x)-u_{i}(z)+\delta\left(u_{i}(x)-u_{i}(y)\right) \\
& \frac{V_{i}(y \mid \sigma)-V_{i}(z \mid \sigma)}{1-\delta}=u_{i}(y)-u_{i}(z)+\delta\left(u_{i}(x)-u_{i}(y)\right) .
\end{aligned}
$$

Thus, optimal voting between $x$ and $y$ is determined by comparing stage payoffs. The difference $V_{i}(x \mid \sigma)-V_{i}(z \mid \sigma)$ is automatically positive for the agenda setter, and for players 2 and 3 , it has the same sign as

$$
u_{i}(x)-\frac{\delta u_{i}(y)+u_{i}(z)}{1+\delta}
$$

and for $\delta$ close to one, this is negative for players 2 and 3 . The difference $V_{i}(y \mid \sigma)-V_{i}(z \mid \sigma)$ is automatically positive for the agenda setter, and for players 2 and 3 , it has the same sign as

$$
\delta u_{i}(x)-u_{i}(z)+(1-\delta) u_{i}(y)
$$

and for $\delta$ close to one, this is positive for player 2 and negative for player 3 .

It follows that voting strategies satisfy condition (ii) in the definition of stationary bargaining equilibrium, and it is evident that the agenda setter's proposal strategy is optimal, satisfying condition (i) in the definition of stationary bargaining equilibrium. We conclude that $\sigma$ is a stationary bargaining equilibrium. 